\title{
Comparison of the infectivity of Trypanosoma cruzi insect-derived metacyclic trypomastigotes after mucosal and cutaneous contaminative challenges
}

\author{
Christopher Steven Eickhoff', Brian Anthony Dunn', Nicole Lea Sullivan ${ }^{1,2}$, Daniel Fredric Hoft ${ }^{1,2 /+}$
}

${ }^{1}$ Department of Internal Medicine ${ }^{2}$ Department of Molecular Microbiology, Saint Louis University, Saint Louis, MO, USA

Trypanosoma cruzi infects humans when infected triatomine vector excreta contaminate breaks in skin or mucosal surfaces. T. cruzi insect-derived metacyclic trypomastigotes (IMT) invade through gastric mucosa after oral challenges without any visible inflammatory changes, while cutaneous and conjunctival infections result in obvious local physical signs. In this study we compared the infectivity of T. cruzi IMT in mice after cutaneous and oral contaminative challenges simulating natural infections. The $50 \%$ infective dose $\left(I D_{50}\right)$ for oral challenge was 100 fold lower than the $I D_{50}$ for cutaneous challenge, indicating that oral mucosal transmission is more efficient than cutaneous transmission.

Key words: Trypanosoma cruzi - mucosal immunity - Chagas disease - animal disease models - infection control

Trypanosoma cruzi is the protozoan parasite that causes Chagas disease, responsible for approximately 11 million infections throughout Latin America (CDC 2007). Chagasic heart disease and gastrointestinal mega-syndromes usually occur after decades of chronic asymptomatic $T$. cruzi infection, initiated by either mucosal or systemic routes of vector-borne transmission (Shaw et al. 1969, Gus et al. 1993, WHO 2010). Infective insect-derived metacyclic trypomastigotes (IMT) are excreted onto the surface of mammalian hosts by infected triatomine bugs, initiating infection of open wounds or mucosal tissues. Contaminative cutaneous [e.g., through the triatomine bite site (TBS)]), as well as conjunctival and oral routes of IMT infection are common (DíazUngria 1968). Because of the long delay between initial T. cruzi infection and the development of chagasic pathology, in most human cases the actual route of initial infection cannot be determined. Therefore, the relative proportions of infections due to cutaneous, conjunctival and oral routes of transmission are not known.

We previously developed mouse models of both conjunctival and oral T. cruzi transmission and determined that the infectious dose establishing infection in $50 \%$ $\left(\mathrm{ID}_{50}\right)$ of the animals challenged was approximately 5-20 IMT (Kirchhoff \& Hoft 1990, Giddings et al. 2006). We further identified gastric epithelial invasion as the anatomical route of infection after oral IMT challenge (Hoft et al. 1996). Gastric infection resulted in no obvious external signs of infection in the mouse model, suggesting that if gastric infection is also the anatomical route of infection

doi: $10.1590 / 0074-0276108042013018$

Financial support: NIH (RO1 AI040196) (to DFH)

+ Corresponding author: hoftdf@slu.edu

Received 12 September 2012

Accepted 4 March 2013 after oral challenge in humans, this route of transmission is likely to be underestimated compared with cutaneous and conjunctival infections which lead to easily visible local cutaneous and periorbital chagomas, respectively. In the current work, we have directly compared the relative $\mathrm{ID}_{50}$ of oral and cutaneous T. cruzi challenges simulating natural vector-borne infection through the TBS.

All animal studies were performed in Association for Assessment and Accreditation of Laboratory Animal Care International accredited facilities [National Institute of Health Assurance (A3225-01)].

The Tulahuén strain of $T$. cruzi and BALB/c mice (Harlan Sprague-Dawley Inc, Indianapolis, USA) were used in these experiments. IMT were prepared by allowing T. cruzi infected triatomine bugs (triatomines, Dipetalogaster maximus) to feed on anesthetised mice. Engorged insects were then incubated in glass vials for 3-5 h, excreta pooled and IMT concentration determined by direct haemocytometer count. Unanesthetised mice were challenged orally with IMT atraumatically by placing 10-30 $\mu \mathrm{L}$ of IMT infected triatomine excreta inside the oropharynx with a P20 pipet as previously described (Kirchhoff \& Hoft 1990, Hoft et al. 1996, Schnapp et al. 2002, Hoft \& Eickhoff 2005). For contaminative cutaneous challenges, the posterior necks of mice were shaved with an electric razor the day before challenge. Immediately before contaminative cutaneous challenge, mice were anesthetised by intraperitoneal injection of ketamine $(60 \mathrm{mg} / \mathrm{kg})$ and xylazine $(5 \mathrm{mg} / \mathrm{kg})$. A single puncture mark was placed through the full thickness of the skin with a tuberculin syringe needle and then 10$30 \mu \mathrm{L}$ of infected triatomine excreta was placed directly over the puncture wound. In some experiments, uninfected triatomine insects were placed onto the shaved area, allowed to feed for $10 \mathrm{~min}$, removed and then infected triatomine excreta placed directly over the feeding puncture wound. Mice remained anesthetised for at least several minutes after placement of infected triatomine 
excreta. Infection nine, 14 or 30 days post-challenge was identified by real-time polymerase chain reaction (RT-PCR) with DNA from local gastric and cutaneous tissues, as well as by recovery of viable tissue parasites from draining lymph nodes, splenic tissues and blood samples as described below.

Tissue DNA samples were studied nine-30 days after IMT challenges. Mouse stomachs were removed from mice orally challenged with IMT by cutting oesophageal and pyloric attachments. These organs were opened along the greater curvature and flushed with sterile phosphate buffered saline. Full thickness patches of skin approximately $0.5 \mathrm{~cm} \times 0.5 \mathrm{~cm}$ were removed from the posterior neck of mice previously challenged with the IMT contaminative cutaneous challenge protocol. Gastric and cutaneous tissue DNA samples were purified and T. cruzi specific RT-PCR performed as described previously (Schnapp et al. 2002).

Viable tissue parasitism in different challenge groups was investigated after oral and cutaneous IMT challenges as described previously (Hoft et al. 1996). Briefly, titrations of gastric lymph node cells, cervical lymph node cells and spleen cells harvested nine-30 days after IMT challenge were incubated at $26^{\circ} \mathrm{C}$ for two months in 96 well microtitre plates with $200 \mu \mathrm{L}$ of liver digested-neutralised tryptose broth (LDNT)+ (Camargo 1964, Kirchhoff et al. 1984). Every two weeks wells were inspected by inverted light microscopy for evidence of $T$. cruzi outgrowth.

Heparinised blood $(250 \mu \mathrm{L})$ from individual mice was collected using aseptic technique and used to inoculate 5 $\mathrm{mL}$ of LDNT+ medium. These cultures were incubated for two months at $26^{\circ} \mathrm{C}$ and examined for outgrowth of $T$. cruzi epimastigotes. T. cruzi-specific IgG ELISAs were conducted on serum samples collected 30 days post-IMT challenge as previously described (Eickoff et al. 2011).
Groups of BALB/c mice were challenged orally and cutaneously with 50, 500 and 5,000 IMT in several experiments. Total DNA was extracted from tissues at the predicted sites of cutaneous or mucosal parasite entry from individual mice on days 9 and 14 post-challenge. DNA was extracted from skin at the site where IMT were deposited over the tuberculin syringe puncture mark. DNA was extracted from gastric tissue after oral challenges which we previously identified as the anatomical site of initial mucosal infection after oral IMT challenge (Hoft et al. 1996). These samples were analysed for the presence of T. cruzi DNA by RT-PCR as previously described (Schnapp et al. 2002). Samples purified from cutaneous and gastric tissues did not have inhibitory effects on PCR amplification of purified total T. cruzi DNA (data not shown). In addition, we harvested draining cervical lymph node cells (from cutaneously challenged mice), gastric lymph node cells (from orally challenged mice) and spleen cells (from all mice) and quantified viable parasites recoverable from all of these tissues as described previously (Hoft et al. 1996). Splenic, gastric lymph node and cervical lymph node cells mixed with parasites and plated in our viable tissue culture assays performed as described previously allowed detection of as few as 1-10 blood form trypomastigotes (BFT) (data not shown). As a third method for identifying parasite infection we performed haemocultures on all mice. In-house studies showed that blood spiked with a single BFT reliably resulted in a positive haemoculture test (data not shown). T. cruzi-specific IgG ELISAs were conducted with serum samples from challenged mice. Table I shows the results of three separate experiments comparing oral vs. cutaneous $T$. cruzi challenges. In the first two experiments, all mice challenged orally with 50-5,000 IMT had evidence of successful in-

TABLE I

Trypanosoma cruzi infection after insect-derived metacyclic trypomastigote (IMT) oral and cutaneous contaminative challenges

\begin{tabular}{lccc}
\hline $\begin{array}{l}\text { T. } \text { cruzi } \\
\text { challenge }\end{array}$ & $\begin{array}{c}\text { Experiment } 1 \\
\mathrm{n} / \mathrm{n}\end{array}$ & $\begin{array}{c}\text { Experiment } 2 \\
\mathrm{n} / \mathrm{n}\end{array}$ & $\begin{array}{c}\text { Experiment } 3 \\
\mathrm{n} / \mathrm{n}\end{array}$ \\
\hline 50 IMT PO & $2 / 2$ & $2 / 2$ & $1 / 3$ \\
500 IMT PO & $1 / 1$ & $2 / 2$ & $3 / 3$ \\
5,000 IMT PO & $2 / 2$ & $2 / 2$ & $2 / 3$ \\
50 IMT cut (NP) & $0 / 2$ & $0 / 2$ & $0 / 3$ \\
500 IMT cut (NP) & $0 / 2$ & $0 / 2$ & $1 / 3$ \\
5,000 IMT cut (NP) & $0 / 3$ & $2 / 2$ & $1 / 3$ \\
50 IMT cut (TBS) & ND & ND & $0 / 3$ \\
500 IMT cut (TBS) & ND & ND & $0 / 3$ \\
5,000 IMT cut (TBS) & ND & ND & $0 / 3$ \\
\hline
\end{tabular}

shown are the number of mice infected/number challenged (from 3 separate experiments). To be considered uninfected, mice had to have negative $T$. cruzi specific polymerase chain reaction (PCR), viable T. cruzi outgrowth assays and T. cruzi-specific IgG ELISA. Parasite outgrowth assay results (quantitative parasite outgrowth cultures and haemocultures) matched real-time PCR results and T. cruzi-specific serum IgG ELISA results in $78 \%$ and $88 \%$ of cases, respectively. Mice were harvested nine days after challenge in experiment 1, 14 days after challenge in experiment 2 and 30 days after challenge in experiment 3. cut: cutaneous; ND: not done; NP: needle prick; PO: perorally; TBS: triatomine bite site. 
fection with $T$. cruzi. In contrast with these results, most mice challenged by our contaminative cutaneous route using a tuberculin syringe puncture mark simulating the TBS did not become infected with $T$. cruzi. In fact, in only one of three experiments did mice reliably become infected after cutaneous challenge and only in the highest dose group (5,000 IMT). To be labelled as negative (uninfected), all assays had to show negative results. To be labelled as positive (infected), at least one assay had to be positive. Parasite outgrowth assay results (quantitative parasite outgrowth cultures and haemocultures) matched RT-PCR results and T. cruzi-specific serum IgG ELISA results in $78 \%$ and $88 \%$ of cases, respectively.

Table II demonstrates significant differences in infectivity comparing oral versus cutaneous infection at all three doses used ( $p<0.05$ by Fisher's exact two-tailed tests). These results agree with our earlier estimations of the ID I0 $_{0}$ for oral mucosal IMT challenges (Kirchhoff \& Hoft 1990) and demonstrate that the $\mathrm{ID}_{50}$ for contaminative cutaneous IMT challenge was $\geq 100$ times higher.

It has been shown that components of sand fly saliva can enhance the infectivity of Leishmania promastigotes by having vasodilatory and anticoagulant effects (Titus $\&$ Ribeiro 1988). To rule out the possibility that our needle puncture cutaneous model underestimates the infectivity of $T$. cruzi IMT for contaminative cutaneous challenge, we conducted further experiments placing IMT infected triatomine excreta directly over the TBS immediately after uninfected triatomines had finished feeding on anesthetised mice. The results shown as experiment 3 in Table I demonstrate that triatomine saliva did not enhance the cutaneous infectivity of T. cruzi IMT.

Contaminative inoculation of IMT into the TBS as well as oral routes of vector-borne T. cruzi infection commonly occurs. However, to our knowledge, there has been no direct comparison of the relative infectivities of

\section{TABLE II}

Statistical significance of oral vs. cutaneous Trypanosoma cruzi challenges

\begin{tabular}{lccc}
\hline T. cruzi challenge & $\begin{array}{c}\text { PO } \\
\mathrm{n} / \mathrm{n}\end{array}$ & $\begin{array}{c}\text { cut (NP + TBS }) \\
\mathrm{n} / \mathrm{n}\end{array}$ & $\mathrm{p}$ \\
\hline 50 IMT & $5 / 7$ & $0 / 10$ & 0.003 \\
500 IMT & $6 / 6$ & $1 / 10$ & 0.001 \\
5,000 IMT & $6 / 7$ & $3 / 11$ & 0.0498 \\
\hline
\end{tabular}

the number of mice infected/number challenged and Fisher's exact two-tailed p-values are shown. To be considered uninfected, mice had to have negative $T$. cruzi specific polymerase chain reaction (PCR), viable $T$. cruzi outgrowth assays, and T. cruzi-specific IgG ELISA. Parasite outgrowth assay results (quantitative parasite outgrowth cultures and haemocultures) matched real-time PCR results and T. cruzi-specific serum IgG ELISA results in $78 \%$ and $88 \%$ of cases, respectively. Mice were harvested nine-30 days after insect-derived metacyclic trypomastigote (IMT) challenge. cut: cutaneous; NP: needle prick; PO: perorally; TBS: triatomine bite site. contaminative cutaneous and oral T. cruzi challenges. Our data indicate that $T$. cruzi IMT more efficiently infect through mucosal surfaces than through breaks in the skin. Multiple reports demonstrate that the oral ingestion of $T$. cruzi IMT can lead to human infection and in some cases outbreaks of $T$. cruzi infection linked to oral transmission have been characterised by high lethality (Benchimol Barbosa 2006, Steindel et al. 2008). In addition, $T$. cruzi is an enzootic infection with many species of mammals serving as reservoirs in nature. Most of this salvatic transmission is thought to be acquired by mammals eating T. cruzi-infected triatomine bugs. Therefore the oral route of $T$. cruzi infection is clearly important and may be overall the most important route of vector borne transmission.

We do not believe that the lower $\mathrm{ID}_{50}$ seen for contaminative cutaneous IMT infection is due to a relative inability of $T$. cruzi parasites to replicate within cutaneous tissues. T. cruzi IMT can infect and replicate within virtually all nucleated mammalian cells. T. cruzi IMT cannot penetrate intact stratum corneum. With the TBS being less than $1 \mathrm{~mm}$ in diameter, the probability of IMT present in triatomine excreta gaining access to this small break in the stratum corneum is small. In contrast, both gastric and conjunctival surfaces provide large areas through which IMT can infect.

Our results demonstrate that $T$. cruzi IMT are significantly more infectious after mucosal compared with cutaneous routes of exposure. We recognise that variations in infectivity may occur with different parasite strains expressing distinct parasite proteins including surface glycoproteins such as gp30/gp82 (Cortez et al. 2012). Further research utilising different parasite/mouse strain combinations is needed to address this question. Regardless, our results indicate that future preventative environmental and vaccination approaches should focus on both the prevention of mucosal and systemic T. cruzi infection.

\section{REFERENCES}

Benchimol Barbosa PR 2006. The oral transmission of Chagas disease: an acute form of infection responsible for regional outbreaks. Int J Cardiol 112: 132-133.

Camargo EP 1964. Growth and differentiation in Trypanosoma cruzi: I. Origin of metacyclic trypanosomes in liquid media. Rev Inst Med Trop Sao Paulo 6: 93-100.

CDC - Centers for Disease Control 2007. Blood donor screening for Chagas disease - United States, 2006-2007. MMWR Morb Mortal Wkly Rep 56: 141-143.

Cortez C, Martins RM, Alves RM, Silva RC, Bilches LC, Macedo S, Atayde VD, Kawashita SY, Briones MR, Yoshida N 2012. Differential infectivity by the oral route of Trypanosoma cruzi lineages derived from y strain. PLoS Negl Trop Dis 6: e1804.

Díaz-Ungria C 1968. Estudio experimental del Trypanosoma cruzi en el perro y otros vertebrados. El problema de la transmisión. Kasmera 3: 73-88.

Eickhoff CS, Vasconcelos JR, Sullivan NL, Blazevic A, Bruna-Romero O, Rodrigues MM, Hoft DF 2011. Co-administration of a plasmid DNA encoding IL-15 improves long-term protection of a genetic vaccine against Trypanosoma cruzi. PLoS Negl Trop Dis 5: e983.

Giddings OK, Eickhoff CS, Smith TJ, Bryant LA, Hoft DF 2006. Anatomical route of invasion and protective mucosal immunity 
in Trypanosoma cruzi conjunctival infection. Infect Immun 74: 5549-5560.

Gus I, Molon ME, Bueno AP 1993. Chagas disease-review of 8 simultaneous cases of acute Chagas myocarditis: 25 years later. Arq Bras Cardiol 60: 99-101.

Hoft DF, Eickhoff CS 2005. Type 1 immunity provides both optimal mucosal and systemic protection against a mucosally invasive, intracellular pathogen. Infect Immun 73: 4934-4940.

Hoft DF, Farrar PL, Kratz-Owens K, Shaffer D 1996. Gastric invasion by Trypanosoma cruzi and induction of protective mucosal immune responses. Infect Immun 64: 3800-3810.

Kirchhoff LV, Hieny S, Shiver M, Snary D, Sher A 1984. Cryptic epitope explains the failure of a monoclonal antibody to bind to certain isolates of Trypanosoma cruzi. J Immunol 133: 2731-2735.

Kirchhoff LV, Hoft DF 1990. Immunization and challenge of mice with insect-derived metacyclic trypomastigotes of Trypanosoma cruzi. Parasite Immunol 12: 65-74.
Schnapp AR, Eickhoff CS, Sizemore D, Curtiss III R, Hoft DF 2002. Cruzipain induces both mucosal and systemic protection against Trypanosoma cruzi in mice. Infect Immun 70: 5065-5074.

Shaw J, Lainson R, Fraiha H 1969. Considerações sobre a epidemiologia dos primeiros casos autóctones de doença de Chagas registrados em Belém, Pará, Brasil. Rev Saude Publica 3: 153-157.

Steindel M, Kramer PL, Scholl D, Soares M, de Moraes MH, Eger I, Kosmann C, Sincero TC, Stoco PH, Murta SM, Carvalho-Pinto CJ, Grisard EC 2008. Characterization of Trypanosoma cruzi isolated from humans, vectors and animal reservoirs following an outbreak of acute human Chagas disease in Santa Catarina state, Brazil. Diagn Microbiol Infect Dis 60: 25-32.

Titus RG, Ribeiro JM 1988. Salivary gland lysates from the sand fly Lutzomyia longipalpis enhance Leishmania infectivity. Science 239: $1306-1308$

WHO - World Health Organization 2010. Chagas disease (American trypanosomiasis). Available from: who.int/neglected_diseases/ diseases/chagas/en/. 